\title{
Power Efficient State Estimation Using Multiple Sensors
}

\author{
Alex S. Leong, Subhrakanti Dey, and Jamie S. Evans \\ ARC Special Research Centre for Ultra-Broadband Information Networks (CUBIN) \\ Department of Electrical and Electronic Engineering \\ University of Melbourne, Parkville, Vic. 3010, Australia \\ email: \{asleong,sdey,jse\}@unimelb.edu.au
}

\begin{abstract}
This paper considers state estimation of scalar linear systems using analog amplify and forwarding with multiple sensors, under a multiple access communication scheme. Optimal state estimation can be achieved using a Kalman filter. We show that in many situations, the error covariance decays at a rate of $1 / M$ when the number of sensors $M$ is large. Optimal allocation of transmission powers subject to constraints on the error covariance or sum power is also considered.
\end{abstract}

\section{Introduction}

Wireless sensor networks are collections of sensors which can communicate with each other or to a central node or base station through wireless links. Potential uses include environment and infrastructure monitoring, healthcare and military applications, to name a few. Often these sensors will have limited energy and computational ability which imposes severe constraints on system design, and signal processing algorithms which can efficiently utilise these resources are of great interest.

In recent years there has been a considerable literature on estimation and detection schemes designed specifically for use in wireless sensor networks. Work on detection in wireless sensor networks include [1] which studies the asymptotic optimality of using identical sensors in the presence of energy constraints, and [2] which derives fusion rules for distributed detection in the presence of fading. Parameter estimation or estimation of constant signals is studied in e.g. [3,4] where issues of quantization and optimization of power usage are addressed. Type based methods for detection and estimation of discrete sources are proposed and analyzed in $[5,6]$.

A promising scheme for distributed estimation in sensor networks is analog forwarding, where measure-

This work was supported by the Australian Research Council ments from the sensors are transmitted directly (possibly scaled) to the fusion center without any coding, which is motivated by optimality results on uncoded transmissions in point-to-point links $[7,8]$. Analog forwarding schemes are attractive due to their simplicity as well as the possibility of real-time processing since there is no coding delay. In [9] the asymptotic (large number of sensors) optimality of analog forwarding for estimating an i.i.d. scalar Gaussian process is shown. Analog forwarding with optimal power allocation is studied in [10] and [11] for multi-access and orthogonal schemes respectively. Lower bounds and asymptotic optimality results for estimating independent vector processes, is addressed in [12].

Rather than the i.i.d. processes previously considered, in this paper we address estimation of dynamical systems using analog forwarding of measurements. In particular, we will consider the problem of state estimation of discrete-time scalar linear systems using multiple sensors. As is well known, optimal state estimation of a linear system can be achieved using a Kalman filter. Other work on Kalman filtering in sensor networks include [13] which studied optimal sensor data quantization, and [14], where Kalman filtering using one bit quantized observations is considered and performance is shown to lie within a constant factor of the standard Kalman filter.

The organization of the paper is as follows. Section 2 specifies our model and preliminaries. We investigate the asymptotic behaviour for a large number of sensors $M$ in Section 3, where it is shown that the error covariance decays at the rate $1 / M$, even when the total power is bounded. Power allocation for static channels is considered in Section 4, where we formulate and solve optimization problems for 1) an error covariance constraint and 2) a sum power constraint. Numerical studies are presented in Section 5. Extensions of our work are outlined in Section 6.

\section{Model and preliminaries}




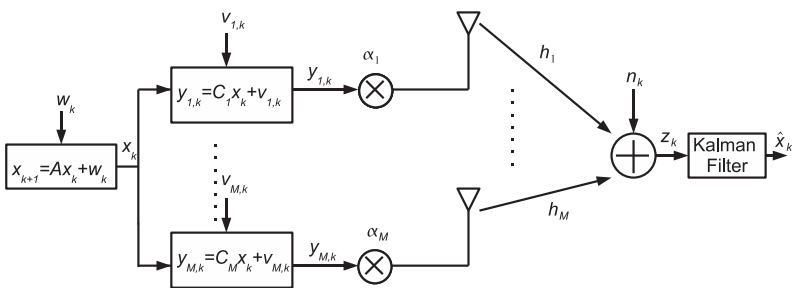

Figure 1: System model

Throughout this paper, $i$ represents the sensor index and $k$ represents the time index. Let the linear system be

$$
x_{k+1}=A x_{k}+w_{k}
$$

with the $M$ sensors each observing

$$
y_{i, k}=C_{i} x_{k}+v_{i, k}, i=1, \ldots, M
$$

with $w_{k}$ and $v_{i, k}$ being zero-mean Gaussians having covariances $Q$ and $R_{i}$ respectively, with the $v_{i, k}$ 's being independent between sensors, see Figure 1. In this paper we will restrict ourselves to scalar systems, so that $A, C_{i}, Q, R_{i}$ are scalar quantities. Moreover, we assume that the system is stable, i.e. $|A|<1$. We consider a multi-access scheme where the fusion center coherently receives the sum

$$
z_{k}=\sum_{i=1}^{M} \alpha_{i} h_{i} y_{i, k}+n_{k}
$$

where $n_{k}$ is zero-mean Gaussian with variance $N, h_{i}$ are the channel gains, and $\alpha_{i}$ are the multiplicative amplification factors in an amplify and forward scheme. The assumption of CSI at the transmitters is crucial in order for the signals to add up coherently in (3), and may not be easy to achieve in large sensor networks. However in studies such as $[15,16]$ it has been shown in slightly different contexts that for moderate amounts of phase error much of the potential performance gains can still be achieved.

The situation (1)-(3) is equivalent to the linear system

$$
\begin{aligned}
x_{k+1} & =A x_{k}+w_{k} \\
z_{k} & =\bar{C} x_{k}+\bar{v}_{k}
\end{aligned}
$$

if we define $\bar{C} \equiv \sum_{i=1}^{M} \alpha_{i} h_{i} C_{i}$ and $\bar{v}_{k} \equiv \sum_{i=1}^{M} \alpha_{i} h_{i} v_{i, k}+$ $n_{k}$, with $\bar{v}_{k}$ having variance $\bar{R} \equiv \sum_{i=1}^{M} \alpha_{i}^{2} h_{i}^{2} R_{i}+N$. Then it is well known that optimal estimation of the state $x_{k}$ in the minimum mean squared error (MMSE) sense can be achieved using a Kalman filter [17].

For stable scalar systems, if $X_{k}$ is stationary we have $\mathbb{E}\left[X_{k}^{2}\right]=\frac{Q}{1-A^{2}}, \forall k$. The power used at time $k$ by the $i$ th sensor in transmitting its measurement to the fusion center is then

$$
p_{i}=\alpha_{i}^{2}\left(C_{i}^{2} \frac{Q}{1-A^{2}}+R_{i}\right) .
$$

From Kalman filtering theory, we know that the steady state $($ as $k \rightarrow \infty)$ error covariance $P_{\infty}$ for scalar systems satisfies

$$
P_{\infty}=\frac{A^{2} P_{\infty} \bar{R}}{\bar{C}^{2} P_{\infty}+\bar{R}}+Q .
$$

The solution to this can be easily shown to be

$$
\frac{\left(A^{2}-1\right) \bar{R}+\bar{C}^{2} Q+\sqrt{\left(\left(A^{2}-1\right) \bar{R}+\bar{C}^{2} Q\right)^{2}+4 \bar{C}^{2} Q \bar{R}}}{2 \bar{C}^{2}} .
$$

It will also be useful to write (5) as

$$
P_{\infty}=\frac{A^{2}-1+Q S+\sqrt{\left(A^{2}-1+Q S\right)^{2}+4 Q S}}{2 S}
$$

with $S \equiv \bar{C}^{2} / \bar{R}$ regarded as a signal-to-noise ratio (SNR). We have the following property, whose proof is omitted for brevity, see [18].

Lemma $1 P_{\infty}$ is a decreasing function of $S$

\section{Asymptotic behaviour}

Since $P_{\infty}$ is a decreasing function of $S$, increasing $S$ will provide an improvement in performance. As $S \rightarrow \infty$, we can see from (6) that $P_{\infty} \rightarrow Q$, the process noise variance. Note that unlike e.g. $[9,11]$ where the mean squared error (MSE) can be driven to zero in some situations such as when there is a large number of sensors, here the lower bound $Q$ on performance is always strictly greater than zero. We will show that as the number of sensors $M \rightarrow \infty$, then $P_{\infty} \rightarrow Q$ can be achieved in many situations. Moreover we will derive the rate $1 / M$ at which this convergence occurs.

In this section we will investigate two simple strategies, 1) $\alpha_{i}=1, \forall i$, and 2) $\alpha_{i}=1 / \sqrt{M}, \forall i{ }^{1}$ We do this first for the "symmetric" case (i.e. the parameters are the same for each sensor) where we can obtain explicit asymptotic expressions. We then use these results to bound the performance in the general asymmetric case. No scaling: $\alpha_{i}=1, \forall i$

Let $\alpha_{i}=1, \forall i$, so measurements are forwarded to the fusion center without any scaling. Assume firstly the

\footnotetext{
${ }^{1}$ These strategies are similar to the case of "equal power constraint" and "total power constraint" in [19] (also [16]), and various versions have also been considered in the work of [9-12], in the context of estimation of i.i.d. processes.
} 
symmetric case, where $C_{i}=C, R_{i}=R, h_{i}=h, \forall i$. We have $\bar{C}=M h C$ and $\bar{v}_{k}$ has variance $\bar{R}=M h^{2} R+N$, so that $S=\frac{M^{2} h^{2} C^{2}}{M h^{2} R+N}$. Substituting into (5):

$$
\begin{aligned}
P_{\infty}= & \frac{1}{2 M^{2} h^{2} C^{2}} \times\left(\left(A^{2}-1\right)\left(M h^{2} R+N\right)+M^{2} h^{2} C^{2} Q\right. \\
& +\left(\left(\left(A^{2}-1\right)\left(M h^{2} R+N\right)+M^{2} h^{2} C^{2} Q\right)^{2}\right. \\
& \left.\left.+4 M^{2} h^{2} C^{2} Q\left(M h^{2} R+N\right)\right)^{1 / 2}\right) .
\end{aligned}
$$

We are interested in the behaviour of this as $M \rightarrow \infty$. Now

$$
\begin{aligned}
(( & \left.\left(A^{2}-1\right)\left(M h^{2} R+N\right)+M^{2} h^{2} C^{2} Q\right)^{2} \\
& \left.+4 M^{2} h^{2} C^{2} Q\left(M h^{2} R+N\right)\right)^{1 / 2} \\
= & \left(h^{4} C^{4} Q^{2} M^{4}+2\left(A^{2}-1\right) R h^{4} C^{2} Q M^{3}\right. \\
& \left.+4 h^{4} C^{2} Q R M^{3}+O\left(M^{2}\right)\right)^{1 / 2} \\
= & h^{2} C^{2} Q M^{2}\left(1+\frac{2\left(A^{2}+1\right) R}{C^{2} Q M}+O\left(\frac{1}{M^{2}}\right)\right)^{1 / 2} \\
= & h^{2} C^{2} Q M^{2}\left(1+\frac{1}{2} \frac{2\left(A^{2}+1\right) R}{C^{2} Q M}+O\left(\frac{1}{M^{2}}\right)\right) \\
= & h^{2} C^{2} Q M^{2}+\left(A^{2}+1\right) h^{2} R M+O(1)
\end{aligned}
$$

where we have used the expansion $(1+x)^{1 / 2}=1+x / 2+$ $O\left(x^{2}\right)$ for $|x|<1$, which is valid when $M$ is sufficiently large. Hence

$$
P_{\infty}=Q+\frac{A^{2} R}{C^{2}} \frac{1}{M}+O\left(\frac{1}{M^{2}}\right)
$$

So in this case the steady state error covariance for the multi-access scheme converges to the process noise variance $Q$, at a rate of $1 / M$. This result matches the rate of $1 / M$ achieved for estimation of i.i.d. processes using multi-access schemes, $[9,12]$, and is also the rate that is achieved for centralised estimation.

Scaling $\alpha_{i}=1 / \sqrt{M}, \forall i$

In the previous case with $\alpha_{i}=1, \forall i$, the power received at the fusion center will grow unbounded as $M \rightarrow \infty$. Suppose instead we let $\alpha_{i}=1 / \sqrt{M}, \forall i$, which will keep the power received at the fusion center bounded (and is constant in the symmetric case), while the transmit power used by each sensor will tend to zero as $M \rightarrow \infty$. Again assume for now that $C_{i}=C, R_{i}=R, h_{i}=h, \forall i$. For this situation we can show similar to $(7)$ that

$$
P_{\infty}=Q+\frac{A^{2}\left(R+N / h^{2}\right)}{C^{2}} \frac{1}{M}+O\left(\frac{1}{M^{2}}\right) .
$$

So we again have the steady state error covariance converging to the process noise variance $Q$ at a rate of
$1 / M$, though the constant in front is larger. The difference here is that the transmit power used by each individual sensor can decrease to zero as the number of sensors increases, which could be quite attractive in power constrained environments such as wireless sensor networks. For i.i.d. processes, this $1 / M$ behaviour when the total received power is bounded has also been observed [12].

General parameters

The behaviour shown in the two previous cases can still hold under more general conditions on $C_{i}, R_{i}$ and $h_{i}$. Suppose for instance that they can be bounded from both above and below, i.e.

$$
\begin{gathered}
0<C_{\min } \leq\left|C_{i}\right| \leq C_{\max }<\infty, \forall i \\
0<R_{\min } \leq R_{i} \leq R_{\max }<\infty, \forall i \\
0<h_{\min } \leq h_{i} \leq h_{\max }<\infty, \forall i
\end{gathered}
$$

We have $M h_{\min } C_{\min } \leq \sum_{i=1}^{M} h_{i} C_{i} \leq M h_{\max } C_{\max }$ and $M h_{\text {min }}^{2} R_{\text {min }} \leq \sum_{i=1}^{M} h_{i}^{2} R_{i} \leq M h_{\text {max }}^{2} R_{\text {max }}$. Recall from Lemma 1 that $P_{\infty}$ is a decreasing function of $S=$ $\bar{C}^{2} / \bar{R}$. If we choose $\alpha_{i} \in\{+1,-1\}$ such that $\alpha_{i} C_{i}$ is positive for all $i$, we have

$$
\frac{M h_{\min }^{2} R_{\min }+N}{M^{2} h_{\max }^{2} C_{\max }^{2}} \leq \frac{\bar{R}}{\bar{C}^{2}} \leq \frac{M h_{\max }^{2} R_{\max }+N}{M^{2} h_{\min }^{2} C_{\min }^{2}}
$$

and by a similar calculation to (7) we can show that as $M \rightarrow \infty$

$$
\begin{aligned}
Q & +\frac{A^{2} h_{\min }^{2} R_{\min }}{h_{\max }^{2} C_{\max }^{2}} \frac{1}{M}+O\left(\frac{1}{M^{2}}\right) \leq P_{\infty} \\
& \leq Q+\frac{A^{2} h_{\max }^{2} R_{\max }}{h_{\min }^{2} C_{\min }^{2}} \frac{1}{M}+O\left(\frac{1}{M^{2}}\right)
\end{aligned}
$$

If instead we choose $\alpha_{i} \in\{1 / \sqrt{M},-1 / \sqrt{M}\}$ such that $\alpha_{i} C_{i}$ is positive for all $i$, and we can similarly show that as $M \rightarrow \infty$

$$
\begin{aligned}
Q & +\frac{A^{2}\left(h_{\min }^{2} R_{\min }+N\right)}{h_{\max }^{2} C_{\max }^{2}} \frac{1}{M}+O\left(\frac{1}{M^{2}}\right) \leq P_{\infty} \\
& \leq Q+\frac{A^{2}\left(h_{\max }^{2} R_{\max }+N\right)}{h_{\min }^{2} C_{\min }^{2}} \frac{1}{M}+O\left(\frac{1}{M^{2}}\right) .
\end{aligned}
$$

In either case, as the upper and lower bounds both converge to $Q$ at a rate of $1 / M, P_{\infty}$ itself will also do so.

\section{Optimal power allocation}

When there are a large number of sensors, one can use simple strategies such as $\alpha_{i}=1 / \sqrt{M}, \forall i$ which will give a convergence of the steady state error covariance to $Q$ at a rate of $1 / M$, while bounding the total power 
used by all the sensors. But when the number of sensors is small, one may perhaps do better with different choices of the $\alpha_{i}$ 's. Here we will address two relevant optimization problems.

\section{Minimizing sum power}

One possible formulation is to minimize the sum of transmit powers used by the sensors subject to a bound on the steady state error covariance, i.e.

$$
\min \sum_{i=1}^{M} p_{i}=\sum_{i=1}^{M} \alpha_{i}^{2}\left(\frac{C_{i}^{2} Q}{1-A^{2}}+R_{i}\right) \text { s.t. } P_{\infty} \leq D
$$

with $P_{\infty}$ given by (5). Some manipulations show that the constraint can be simplified to $\bar{R}\left(A^{2} D+Q-D\right)+$ $\bar{C}^{2} D(Q-D) \leq 0$. Now define $s=h_{1} C_{1} \alpha_{1}+\cdots+$ $h_{M} C_{M} \alpha_{M}$. Then the optimization problem becomes equivalent to

$$
\begin{aligned}
& \min _{\alpha_{1}, \ldots, \alpha_{M}, s} \sum_{i=1}^{M} \alpha_{i}^{2}\left(\frac{C_{i}^{2} Q}{1-A^{2}}+R_{i}\right) \\
& \text { s.t. }\left(\sum_{i=1}^{M} \alpha_{i}^{2} h_{i}^{2} R_{i}+N\right)\left(A^{2} D+Q-D\right) \leq s^{2} D(D-Q) \\
& \text { and } s=\sum_{i=1}^{M} h_{i} C_{i} \alpha_{i} .
\end{aligned}
$$

Before going further, let us determine some upper and lower bounds on $D$. From Section 3, a lower bound is $D \geq Q$, where $Q$ is the process noise variance. For an upper bound, suppose $\bar{C}=0$ so we don't have any information about $x_{k}$. Since we are assuming the system is stable, one can still achieve an error covariance of $\frac{Q}{1-A^{2}}$ (just let $\left.\hat{x}_{k}=0, \forall k\right)$, so $D \leq \frac{Q}{1-A^{2}}$. Hence in problem (11) both $D-Q \geq 0$ and $A^{2} D+Q-D \geq 0$.

The objective function of problem (11) is clearly convex. Let us call $x=A^{2} D+Q-D, y=D(D-$ $Q), a_{i}=h_{i} C_{i}, b_{i}=h_{i}^{2} R_{i}$ and $\gamma_{i}=\left(\frac{C_{i}^{2} Q}{1-A^{2}}+R_{i}\right)$ for $i=1, \ldots, M$. We can divide the feasible region into two regions corresponding to $s>0$ and $s<0$. Then in each of the two regions, the function $\left(\sum_{i=1}^{M} \alpha_{i}^{2} b_{i}+\right.$ $N) / s^{2}$ is convex, by noting that each of the functions $\alpha_{i}^{2} / s^{2}$ is convex. Hence the two regions corresponding to $s>0$ and $s<0$ are both convex and the global solution can be easily found numerically. Moreover, following similar steps to [10], a solution in (mostly) closed form can be obtained. We omit the derivations but shall summarise what is required. One first solves numerically for $\lambda$ the equation

$$
\sum_{i=1}^{M} \frac{\lambda a_{i}^{2}}{\gamma_{i}+\lambda b_{i} x}=\frac{1}{y}
$$

Since the left hand side is increasing with $\lambda$ solutions to this equation will be unique provided it exists. Taking limits as $\lambda \rightarrow \infty$, we see that a solution exists if and only if

$$
\sum_{i=1}^{M} \frac{a_{i}^{2}}{b_{i}}>\frac{x}{y}
$$

Equation (12) thus also provides a feasibility check for the optimization problem (11). In the context of (11), one can easily derive that (12) implies

$$
\sum_{i=1}^{M} \frac{C_{i}^{2}}{R_{i}}>\frac{A^{2} D+Q-D}{D(D-Q)}
$$

which indicates that the sum of the sensor signal to noise ratios must be greater than a threshold (dependent on the error covariance threshold $D$ ) for the optimization problem (11) to be feasible.

Next, we compute $\mu$ from

$$
\mu^{2}=N x\left(\sum_{i=1}^{M} \frac{a_{i}^{2} \gamma_{i}}{4 \lambda\left(\gamma_{i}+\lambda b_{i} x\right)^{2}}\right)^{-1}
$$

Finally we obtain the optimal $\alpha_{i}$ 's (denoted by $\alpha_{i}^{*}$ )

$$
\alpha_{i}^{*}=\frac{\mu a_{i}}{2\left(\gamma_{i}+\lambda b_{i} x\right)}, i=1, \ldots, M
$$

with the resulting powers

$$
p_{i}=\alpha_{i}^{* 2} \gamma_{i}=\alpha_{i}^{* 2}\left(C_{i}^{2} \frac{Q}{1-A^{2}}+R_{i}\right), i=1, \ldots, M .
$$

Depending on whether we choose $\mu$ to be positive or negative, two different sets of $\alpha_{i}^{*}$ 's will be obtained, one of which is the negative of the other, though the $p_{i}$ 's and hence the optimal value of the objective function remains the same.

We remark also that this problem can be solved in a distributed manner, where the fusion center can calculate the values $\lambda$ and $\mu$ and broadcast them to all sensors, which can then be used by the sensors along with their local information to determine the optimal $\alpha_{i}$ 's, see $[10]$.

\section{Minimizing error covariance}

A related problem is to minimize the steady state error covariance subject to a sum power constraint. Formally, this is

$$
\min P_{\infty} \text { s.t. } \sum_{i=1}^{M} \alpha_{i}^{2}\left(\frac{C_{i}^{2} Q}{1-A^{2}}+R_{i}\right) \leq p_{\text {total }}
$$

with $P_{\infty}$ again given by (5). By Lemma 1 , maximizing $\bar{C}^{2} / \bar{R}$ (or minimizing $\bar{R} / \bar{C}^{2}$ ) is equivalent to minimiz- 
ing $P_{\infty}$. Hence the problem is equivalent to

$$
\begin{aligned}
& \min _{\alpha_{1}, \ldots, \alpha_{M}, s} \frac{\sum_{i=1}^{M} \alpha_{i}^{2} h_{i}^{2} R_{i}+N}{s^{2}} \\
& \text { s.t. } \sum_{i=1}^{M} \alpha_{i}^{2}\left(\frac{C_{i}^{2} Q}{1-A^{2}}+R_{i}\right) \leq p_{\text {total }} \text { and } s=\sum_{i=1}^{M} h_{i} C_{i} \alpha_{i}
\end{aligned}
$$

which can also be shown to be convex, though unlike problem (11), we have not been able to obtain an analytical solution here.

\section{Numerical studies}

First we show some plots for the asymptotic results of Section 3. In Fig. 2(a) we plot $P_{\infty}$ vs $M$ for the symmetric situation with $\alpha_{i}=1 / \sqrt{M}$ and $A=0.8, Q=$ $1.5, N=1, C=1, R=1, h=0.8$. We compare this with the asymptotic expression $Q+\frac{A^{2}\left(R+N / h^{2}\right)}{C^{2}} \frac{1}{M}$ from (9). Fig. 2(b) plots the difference between $P_{\infty}-Q$, and compares this with the term $\frac{A^{2}\left(R+N / h^{2}\right)}{C^{2}} \frac{1}{M}$. We can see that $P_{\infty}$ is well approximated by the asymptotic expression even for 20-30 sensors.
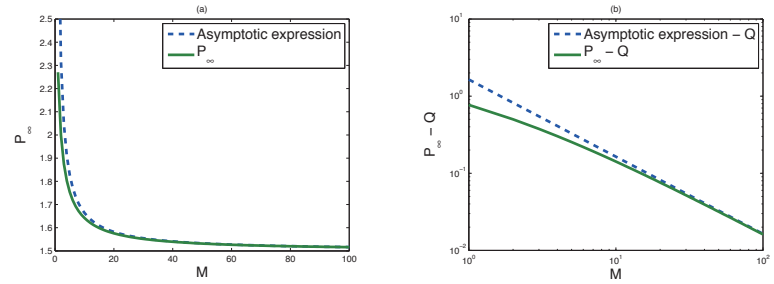

Figure 2: Comparison between $P_{\infty}$ and asymptotic expression: $\alpha_{i}=1 / \sqrt{M}$

Next we look at numerical results for optimal power allocation in Section 4. In Figure 3 we compare between using optimal power allocation and a simple equal power allocation scheme. We use $A=0.9, N=1, Q=$ 1 with various values for $C_{i}, R_{i}$ drawn from a uniform distribution $U(0,2)$, and values of $h_{i}$ drawn from $U(0,1)$. In (a) we keep $D=2$, while in (b) we keep $p_{\text {total }}=1$. What can be observed is that as the number of sensors $M$ increases there is a general trend downwards for both graphs, though for equal power allocation the behaviour is not necessarily monotonic. This is due to the fact that some sensors might have low quality measurements, e.g. sensor 5 , so that extra resources are needed to compensate.

\section{Extensions}

In this section we briefly outline some extensions of the work in this paper. Further details can be found in $[18]$.
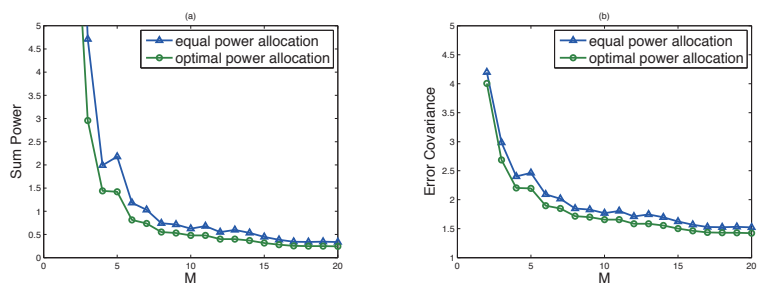

Figure 3: Comparison between power allocation schemes, with (a) an error covariance constraint and (b) a sum power constraint

\subsection{Fading channels}

Instead of (3), we can allow the channels gains $h_{i, k}$ to be time varying as follows:

$$
z_{k}=\sum_{i=1}^{M} \alpha_{i, k} h_{i, k} y_{i, k}+n_{k}
$$

where the amplification factors $\alpha_{i, k}$ are now also assumed to be time varying.

Using the shorthand $P_{k}=P_{k \mid k-1}$, the Kalman filter recursion for the error covariance in the case where CSI is available is (see [17]):

$$
P_{k+1}=A^{2} P_{k}-\frac{A^{2} P_{k}^{2} \bar{C}_{k}^{2}}{\bar{C}_{k}^{2} P_{k}+\bar{R}_{k}}+Q=\frac{A^{2} P_{k} \bar{R}_{k}}{\bar{C}_{k}^{2} P_{k}+\bar{R}_{k}}+Q
$$

with $\bar{C}_{k} \equiv \sum_{i=1}^{M} \alpha_{i, k} h_{i, k} C_{i}$ and $\bar{R}_{k} \equiv \sum_{i=1}^{M} \alpha_{i, k}^{2} h_{i, k}^{2} R_{i}+$ $N$. One way in which we can formulate an optimization problem is to minimize the sum of powers used at each time instant, subject to $P_{k+1 \mid k} \leq D$ at all time instances $k$. Another possible optimization problem is to minimize $P_{k+1 \mid k}$ at each time instant subject to a sum power constraint at each time $k$. These problems will be of a similar form to those treated in Section 4.

In the case where CSI is not available but channel statistics are available, one can instead derive the best linear estimator similar to [20], and its performance can be analyzed similarly.

\subsection{Orthogonal access scheme}

Another extension is to use an orthogonal access scheme, such as considered in [11]. Here instead of the sum (3), the sensors each transmit via orthogonal channels, so that the fusion center receives

$$
z_{i, k}=\alpha_{i, k} h_{i, k} y_{i, k}+n_{i, k}, i=1, \ldots, M .
$$

For time-invariant channels, the steady state error covariance can be shown to be

$$
P_{\infty}^{o}=\frac{A^{2}-1+Q S^{o}+\sqrt{\left(A^{2}-1+Q S^{o}\right)^{2}+4 Q S^{o}}}{2 S^{o}}
$$


with $S^{o} \equiv \sum_{i=1}^{M} \frac{\alpha_{i}^{2} h_{i}^{2} C_{i}^{2}}{\alpha_{i}^{2} h_{i}^{2} R_{i}+N}$. Asymptotic behaviour of the error covariance and optimal power allocation can also be done for this scheme, and compared with the multi-access scheme considered in this paper.

\section{Conclusion}

This paper has investigated the use of analog forwarding in the distributed estimation of stable scalar linear systems. Under a multi-access scheme, we have shown a $1 / M$ scaling behaviour of the error covariance in a number of different situations, and have also formulated and solved some optimal power allocation problems.

\section{References}

[1] J.-F. Chamberland and V. V. Veeravalli, "Decentralized detection in sensor networks," IEEE Trans. Signal Processing, vol. 51, no. 2, pp. 407416, Feb. 2003.

[2] B. Chen, R. Jiang, T. Kasetkasem, and P. K. Varshney, "Channel aware decision fusion in wireless sensor networks," IEEE Trans. Signal Processing, vol. 52, no. 12, pp. 3454-3458, Dec. 2004.

[3] A. Ribeiro and G. B. Giannakis, "Bandwidthconstrained distributed estimation for wireless sensor networks, Part I: Gaussian case," IEEE Trans. Signal Processing, vol. 54, no. 3, pp. 11311143, Mar. 2006.

[4] J.-J. Xiao, S. Cui, Z.-Q. Luo, and A. J. Goldsmith, "Power scheduling of universal decentralized estimation in sensor networks," IEEE Trans. Signal Processing, vol. 54, no. 2, pp. 413-422, Feb. 2006.

[5] G. Mergen and L. Tong, "Type based estimation over multiaccess channels," IEEE Trans. Signal Processing, vol. 54, no. 2, pp. 613-626, Feb. 2006.

[6] K. Liu and A. M. Sayeed, "Type-based decentralized detection in wireless sensor networks," IEEE Trans. Signal Processing, vol. 55, no. 5, pp. 1899 1910, May 2007.

[7] T. J. Goblick, "Theoretical limitations on the transmission of data from analog sources," IEEE Trans. Inform. Theory, vol. 11, no. 4, pp. 558-567, Oct. 1965.

[8] M. Gastpar, B. Rimoldi, and M. Vetterli, "To code, or not to code: Lossy source-channel communication revisited," IEEE Trans. Inform. Theory, vol. 49, no. 5, pp. 1147-1158, May 2003.
[9] M. Gastpar and M. Vetterli, "Source-channel communication in sensor networks," Springer Lecture Notes in Computer Science, vol. 2634, pp. 162177, Apr. 2003.

[10] J.-J. Xiao, Z.-Q. Luo, S. Cui, and A. J. Goldsmith, "Power-efficient analog forwarding transmission in an inhomogeneous Gaussian sensor network," in Proc. SPAWC, New York City, NY, June 2005.

[11] S. Cui, J.-J. Xiao, A. Goldsmith, Z.-Q. Luo, and H. V. Poor, "Estimation diversity and energy efficiency in distributed sensing," IEEE Trans. Signal Processing, vol. 55, no. 9, pp. 4683-4695, Sept. 2007.

[12] M. Gastpar and M. Vetterli, "Power, spatiotemporal bandwidth, and distortion in large sensor networks," IEEE J. Select. Areas Commun., vol. 23, no. 4, pp. 745-754, Apr. 2005.

[13] K. Zhang and X. R. Li, "Optimal sensor data quantization for best linear unbiased estimation fusion," in Proc. IEEE Conf. Decision and Control, Atlantis, Bahamas, Dec. 2004, pp. 2656-2661.

[14] A. Ribeiro, G. B. Giannakis, and S. I. Roumeliotis, "SOI-KF: Distributed Kalman filtering with low-cost communications using the sign of innovations," IEEE Trans. Signal Processing, vol. 54, no. 12, pp. 4782-4795, Dec. 2006.

[15] R. Mudumbai, G. Barriac, and U. Madhow, "On the feasibility of distributed beamforming in wireless networks," IEEE Trans. Wireless Commun., vol. 6, no. 5, pp. 1754-1763, May 2007.

[16] W. Li and H. Dai, "Distributed detection in wireless sensor networks using a multiple access channel," IEEE Trans. Signal Processing, vol. 55, no. 3, pp. 822-833, Mar. 2007.

[17] B. D. O. Anderson and J. B. Moore, Optimal Filtering. New Jersey: Prentice Hall, 1979.

[18] [Online].

Available: http://arxiv.org/abs/0803.3850

[19] K. Liu, H. El Gamal, and A. M. Sayeed, "Decentralized inference over multiple-access channels," IEEE Trans. Signal Processing, vol. 5, no. 7, pp. 3445-3455, July 2007.

[20] P. K. Rajasekaran, N. Satyanarayana, and M. D. Srinath, "Optimum linear estimation of stochastic signals in the presence of multiplicative noise," IEEE Trans. Aerosp. Electron. Syst., vol. 7, no. 3, pp. 462-468, May 1971. 